\title{
Confinement and manipulation of atoms using short laser pulses
}

\author{
T.G.M. Freegarde ${ }^{1}$, J. Walz, T.W. Hänsch \\ Max Planck Institut für Quantenoptik, Hans-Kopfermann-Straße 1, D-85748 Garching bei München, Germany
}

Received 16 February 1995

\begin{abstract}
The force resulting from a position-dependent sequence of interactions with short counter-propagating $\pi$-pulses of laser radiation can propel atoms towards the small region where the pulses overlap. The optical trap thus formed may be combined with Doppler-cooling laser beams.
\end{abstract}

\section{Introduction}

The trapping and cooling of atomic and ionic samples are now established techniques in high resolution spectroscopy. Laser cooling methods [1] require only the illumination of the sample with suitable beams of light, and allow the thermal motion of the atoms to be essentially eliminated, reducing the Doppler shift and increasing the time for which an atom may be observed. Electromagnetic trapping schemes [2] meanwhile confine the sample from preparation through to measurement but use (quasi-) static electric and magnetic fields, requiring the presence near the sample of pole pieces and electrodes, and incurring Stark and Zeeman shifts and splittings. Whilst a trap having the simple convenience of laser cooling would be highly desirable, stable confinement using just the strong scattering force of a steady light field may be shown to be impossible [3]. Optical traps, as opposed to molasses, therefore use Zeeman-tuning [4] or optical pumping [5] to introduce an additional positiondependence, or work with the weaker optical gradient (dipole) force [6].

\footnotetext{
' Present address: Physical Chemistry Laboratory, Oxford University, South Parks Road, Oxford OX1 3QZ, UK.
}

In this paper, we propose an alternative scheme in which counter-propagating beams of short pulses of light, as from a mode-locked laser, are used to provide a position-dependent scattering force towards a volume where the pulses meet. Stable confinement using the strong scattering force of a resonant laser field is possible because the interaction - like that in the Paul trap - is time-dependent. Whereas the instantaneous fields of the Paul trap are themselves positiondependent, however, the force described here depends fundamentally upon the temporal variation of the laser pulses, and derives not only from the instantaneous field, but also from the history of the light fields and the atom thereby prepared. Because of the finite speed of light this leads to a position-dependent potential, which is thus determined by the spatial variation, in the direction of propagation, of the light pulses themselves.

\section{Principle of operation}

The principle of our confinement scheme is as follows. The atomic sample is irradiated from two opposite directions by a stream of pulses from a modelocked laser, perhaps with a typical duration of a pi- 
cosecond and a repetition rate of order $10-100 \mathrm{MHz}$, and tuned to an atomic transition. Each pulse is a socalled " $\pi$ "-pulse, meaning that the probability of absorption (or of stimulating emission from the excited atom) is unity, and the beams are arranged so that at the centre of the sample region, two pulses will arrive simultaneously ${ }^{2}$. For an atom displaced somewhat from the trap centre, however, the two pulses will be separated in time: the atom will first be excited, by a pulse whose absorbed momentum will direct it towards the centre; in contrast, the second, counterpropagating, $\pi$-pulse will stimulate the emission from the excited atom of a photon in the opposite direction to that initially absorbed. The two pulses accordingly leave the atom in its initial, unexcited state, but with two units of photon momentum towards the centre of the trap, whilst a photon from the first pulse is scattered into the second, counter-propagating beam. The force is therefore position dependent, unlike the Doppler-cooling force which depends upon the velocity of the atom; indeed, the broad spectral width of the short light pulses ensures that Doppler-cooling cannot here occur.

With non- $\pi$-pulses, and hence non-unity transition probability, a smaller restoring force will still on average be produced (although with higher intensities a stronger force may exist near the centre). The atom may be left, after the passage of the two pulses, in the excited state, in which case the next pulse-pair will have a negative, repelling effect. If, however, the period between successive pulses is greater than the lifetime of the excited state, spontaneous emission will ensure that the atom enters each cycle in the ground state, as required. This condition, that atomic coherence is lost between successive pulses, requires that the mode-spacing of the pulsed laser be less than the spectral width of the atomic transition, and hence that no velocity-selective effects may occur. The maximum acceleration is thus limited to that with saturated Doppler cooling. Spontaneous emission will, of course, lead to heating of the atomic sample.

Within the region of overlap of the two pulses, perhaps a millimetre thick, the process is also less well defined. The counter-propagating pulses will interfere

\footnotetext{
${ }^{2}$ A number of schemes have been suggested for deflecting or cooling atoms using $\pi$-pulses. The interested reader is referred to Ref. [7].
}

and the excitation probability may once again depart from unity, whilst the directions of photon absorption and stimulated emission are no longer unique. Multiphoton processes (diffraction of atoms by the standing wave) and spontaneous emission make the impulse of each pulse pair uncertain. Nonetheless, thermal motion corresponds to many times the impulse momentum and the confinement volume will extend over many optical wavelengths, so the stochastic nature of the overlap-region impulses may be well represented by averaged force and heating terms for all but the slowest atoms. In the absence of additional cooling mechanisms, the heating at the trap centre will limit the time for which atoms may be confined.

\section{Analysis for a two-level atom}

\subsection{Outside the trap centre}

Our analysis, for a two-level system, first considers the situation away from the trap centre. If an atom, initially in state $i$, is illuminated for a time $t$ with radiation of intensity $I$ which is resonant with the transition $i \rightarrow j$, then the probability of excitation to the state $j$, neglecting spontaneous decay, is given by

$P_{i j}=\sin ^{2} \frac{\Omega t}{2}$,

where $\Omega$ is the Rabi frequency given by

$\Omega^{2}=\frac{3 \lambda^{3}}{2 \pi h c} I \frac{g_{j}}{g_{i}} A_{j i}$

where $\lambda, g_{i, j}$ and $A_{j i}$ are the wavelength, degeneracies and probability of the atomic transition. It follows that a rectangular " $\pi$-pulse", giving unity excitation probability, has an intensity $I_{\pi}$ and duration $t_{\pi}$ related by

$I_{\pi}=\frac{2}{3} \pi^{3} h c \frac{g_{i}}{g_{j} A_{j i} \lambda^{3}} \frac{1}{t_{\pi}^{2}}$

If the atom is sufficiently displaced (i.e. $>\frac{1}{2} c t_{\pi}$ ) from the trap centre for the two counter-propagating pulses to be separated distinctly in time, then the atom will receive, in one absorption and stimulated emission cycle, the full impulse of two photon momenta

$\Delta p=2 h k=2 h / \lambda$. 
Consequently, if the atomic momentum changes by $\Delta p$ with each pulse from the laser, the force experienced is

$F=(2 h / \lambda) f$,

where $f$ is the pulse repetition frequency. The twophoton recoil velocity $v_{\text {rec }}=2 h / m \lambda$ follows similarly from Eq. (4); the ratio of the recoil velocity to the r.m.s. thermal velocity $v_{\mathrm{th}}=\sqrt{3 k T / m}$ is typically of order $10^{-4}$ for visible wavelengths and room temperature.

The constant force outside the overlap region leads to a linearly varying potential, whose depth is equal to the characteristic thermal energy $\frac{3}{2} k_{\mathrm{B}} T$ at a displacement $x_{\text {th }}=3 k T \lambda / 2 h f$, which is typically a few centimetres at room temperature.

\subsection{Within the overlap region}

Outside the overlap region, the atom interacts at any time with only one of the laser beams, and after the first pulse has passed there is a unique correspondence betwecn the probability of excitation and the impulse received by the atom. If the first pulse is an exact $\pi$ pulse, the overall change in momentum resulting from an interaction with the pulse pair is then defined. In the region where the pulses overlap, however, an excited atom could have absorbed with some probability from either beam; furthermore, multi-photon processes are possible, an examplc being a double excitation from one direction separated by stimulated emission into the opposite beam, yielding a net impulse of $3 \hbar k$.

The deflection of an atomic beam by an optical standing wave has already been considered in much detail [8], but corresponds to our arrangement only at the centre of the trap, where the system is symmetrical and no net force cxists. In the following calculations, therefore, we permit a relative delay between the two counter-propagating pulses.

In order to calculate the behaviour within the overlap region, we use a bare-state representation of the atom and slowly-varying radiation-field, which we label according to the atomic state $g$ or $e$, the photon number in the positive propagation direction $n_{+}$and the photon number in the negative direction $n_{-}$. Before interaction, the system state will thus be $\left|g, n_{+}(0), n_{-}(0)\right\rangle$. Since the total momentum is conserved and we consider only a two-level atom, the states may also be labelled uniquely and more usefully according to the change in atomic momentum $p$ :

$$
\begin{aligned}
& \left|g, n_{+}+1, n_{-}-1\right\rangle \ldots|-2\rangle \\
& \left|e, n_{+}, n_{-}-1\right\rangle \ldots|-1\rangle \\
& \left|g, n_{+}, n_{-}\right\rangle \ldots|0\rangle \\
& \left|e, n_{+}-1, n_{-}\right\rangle \ldots|+1\rangle \\
& \left|g, n_{+}-1, n_{-}+1\right\rangle \ldots|+2\rangle \\
& \left|e, n_{+}-2, n_{-}+1\right\rangle \ldots|+3\rangle
\end{aligned}
$$

The interaction Hamiltonian for an atom and radiation field may be written, in the Schrödinger representation and subject to the rotating-wave approximation, as [9]

$$
\begin{aligned}
& \hat{\mathcal{H}}_{\mathrm{ED}}=\mathrm{i} e \sum_{\boldsymbol{k}}\left(\hbar \omega_{\boldsymbol{k}} / 2 \epsilon_{0} V\right)^{1 / 2} \hat{\boldsymbol{\varepsilon}}_{k} \cdot \boldsymbol{D}_{i j} \\
& \quad \times\left\{\hat{\boldsymbol{\pi}}^{\dagger} \hat{a}_{\boldsymbol{k}} \exp (\mathrm{i} \boldsymbol{k} \cdot \boldsymbol{R})-\hat{a}_{\boldsymbol{k}}^{\dagger} \hat{\boldsymbol{\pi}} \exp (-\mathrm{i} \boldsymbol{k} \cdot \boldsymbol{R})\right\},
\end{aligned}
$$

where $V$ is the mode volume, $D_{i j}$ is the atomic dipole matrix element, and the radiation field components are defined by their wavevector $k$, frequency $\omega_{k}$, and polarization $\hat{\boldsymbol{\varepsilon}}_{k}$. In our case, we have two atomic levels $|g\rangle$ and $|e\rangle$, and two opposite propagation directions for beams of wavevectors $\boldsymbol{k}$ and $-\boldsymbol{k}$, whilst we represent the two light fields by the initial photon numbers $n_{+}(t-k \cdot R / \omega)$ and $n_{-}(t+k \cdot R / \omega)$ for the positive- and negative-travelling pulses. The interaction Hamiltonian in our case is therefore

$$
\begin{gathered}
\hat{\mathcal{H}}_{\mathrm{ED}}=\mathrm{i} e \sqrt{\frac{\hbar \omega}{2 \epsilon_{0} V}}\left[\hat{\boldsymbol{\varepsilon}}_{+} \cdot D_{g e}\left\{\hat{\pi}^{\dagger} \hat{a}_{+} \mathrm{e}^{\mathrm{i} \boldsymbol{k} \cdot \boldsymbol{R}}-\hat{a}_{+}^{\dagger} \hat{\pi} \mathrm{e}^{-\mathrm{i} \boldsymbol{k} \cdot \boldsymbol{R}}\right\}\right. \\
\left.+\hat{\boldsymbol{\varepsilon}}_{-} \cdot \boldsymbol{D}_{g e}\left\{\hat{\pi}^{\dagger} \hat{a}_{-} \mathrm{e}^{-\mathrm{i} \boldsymbol{k} \cdot \boldsymbol{R}}-\hat{a}_{-}^{\dagger} \hat{\boldsymbol{\pi}} \mathrm{e}^{\mathrm{i} \boldsymbol{k} \cdot \boldsymbol{R}}\right\}\right]
\end{gathered}
$$

We note that this electric-dipole interaction couples only states involving a change of atomic state and a single increment or decrement of one of the photon states. In the momentum-labelled notation, then, the only non-zero matrix elements are those for which $\Delta p= \pm 1$; such elements belong to one of four possibilities, depending upon the impulse and initial atomic state. If the initial state is the ground state ( $p$ even in our notation), then 


$$
\begin{aligned}
\langle p & \left.+1\left|\mathcal{H}_{\mathrm{ED}}\right| \boldsymbol{p}_{\text {even }}\right\rangle=\mathrm{i} e \sqrt{\frac{\hbar \omega}{2 \epsilon_{0} V}} \\
& \times \hat{\boldsymbol{\varepsilon}}_{+} \cdot \boldsymbol{D}_{g e} \mathrm{e}^{\mathrm{i} \boldsymbol{k} \cdot \boldsymbol{R}} n_{+}(t-\boldsymbol{k} \cdot \boldsymbol{R} / \omega)^{1 / 2}
\end{aligned}
$$

and we note that $\hbar \omega n_{ \pm}(t) / V=I_{ \pm}(t) / c$, where $I_{ \pm}(t)$ are the time-dependent pulse intensities. Since $\boldsymbol{k} \cdot \boldsymbol{R}$ will usually be essentially constant during the interaction with the pulse pair, we may now make the trans-

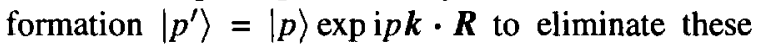
phase-factors from the interaction Hamiltonian, and Eq. 8 becomes

$$
\begin{aligned}
\left\langle\boldsymbol{p}^{\prime}\right. & \left.+1\left|\mathcal{H}_{\mathrm{ED}}\right| \boldsymbol{p}_{\mathrm{even}}^{\prime}\right\rangle \\
& =\frac{\mathrm{i} e}{\left(2 \epsilon_{0} c\right)^{1 / 2}} \hat{\boldsymbol{\varepsilon}}_{+} \cdot D_{g e} I_{+}^{1 / 2}(t-\boldsymbol{k} \cdot \boldsymbol{R} / \omega)
\end{aligned}
$$

and for $\Delta \boldsymbol{p}^{\prime}=-1$,

$$
\begin{aligned}
\left\langle\boldsymbol{p}^{\prime}\right. & \left.-1\left|\mathcal{H}_{\mathrm{ED}}\right| \boldsymbol{p}_{\mathrm{even}}^{\prime}\right\rangle \\
= & \frac{\mathrm{i} e}{\left(2 \epsilon_{0} c\right)^{1 / 2}} \hat{\boldsymbol{\varepsilon}}_{-} \cdot \boldsymbol{D}_{g e} I_{-}^{1 / 2}(t+k \cdot R / \omega) .
\end{aligned}
$$

Similarly, if the initial state is the excited state, corresponding to $\boldsymbol{p}^{\prime}$ being odd,

$$
\begin{aligned}
\left\langle\boldsymbol{p}^{\prime}\right. & \left.+1\left|\mathcal{H}_{\mathrm{ED}}\right| \boldsymbol{p}_{\mathrm{odd}}^{\prime}\right\rangle \\
& =-\frac{\mathrm{i} e}{\left(2 \epsilon_{0} c\right)^{1 / 2}} \hat{\boldsymbol{\varepsilon}}_{-} \cdot \boldsymbol{D}_{g e} I_{-}^{1 / 2}(t+\boldsymbol{k} \cdot \boldsymbol{R} / \omega), \\
\left\langle\boldsymbol{p}^{\prime}\right. & \left.-1\left|\mathcal{H}_{\mathrm{ED}}\right| \boldsymbol{p}_{\mathrm{odd}}^{\prime}\right\rangle \\
& =-\frac{\mathrm{i} e}{\left(2 \epsilon_{0} c\right)^{1 / 2}} \hat{\boldsymbol{\varepsilon}}_{+} \cdot \boldsymbol{D}_{g e} I_{+}^{1 / 2}(t-\boldsymbol{k} \cdot \boldsymbol{R} / \omega) .
\end{aligned}
$$

In order to calculate the momentum change experienced by the atom, we perform numerical integration of the Schrödinger equation

$\mathrm{i} \hbar \frac{\mathrm{d}}{\mathrm{d} t} \Psi=\left(\hat{\mathcal{H}}_{\mathrm{E}}+\hat{\mathcal{H}}_{\mathrm{R}}+\hat{\mathcal{H}}_{\mathrm{ED}}\right) \Psi$,

where the pure electronic and radiation field terms $\left(\hat{\mathcal{H}}_{\mathrm{E}}+\hat{\mathcal{H}}_{\mathrm{R}}\right) \Psi=\left(\mathcal{E}_{\mathrm{E}}+\mathcal{E}_{\mathrm{R}}\right) \Psi$, represent the total energy of the atom-field system, and may be set to zero. Our calculations were performed for rectangular, Gaussian and $\operatorname{sech}^{2} \pi$-pulses of equal energy, the Gaussian and $\operatorname{sech}^{2}$ forms being given by $I=2 I_{\pi} \exp -\left(2 \sqrt{\pi} t / t_{\pi}\right)^{2}$ and $I=$ $\left(\pi^{2} / 4\right) I_{\pi} \operatorname{sech}^{2}\left[\pi^{2} t /\left(2 t_{\pi}\right)\right]$, where $I_{\pi}$ and $t_{\pi}$ are the intensity and duration of the rectangular $\pi$-pulse, defined in Eq. (3).
Heisenberg's uncertainty principle prevents us from measuring a single photon recoil with the atom localized to within an optical wavelength. We therefore take for our calculations the realistic case of measuring the deflection of an atomic beam, and prepare the atomic sample in a specific momentum state whose spatial distribution is broad with respect to the laser wavelength. Starting from an initial state $\Psi=|0\rangle$, we arrive at a final wavefunction

$$
\Psi=\sum_{p^{\prime}} C_{p^{\prime}}\left|p^{\prime}\right\rangle=\sum_{p^{\prime}=p} C_{p^{\prime}} \mathrm{e}^{-\mathrm{i} p k \cdot R}|p\rangle
$$

from which we calculate the mean impulse $\mathcal{P}$, the rms breadth of the impulse distribution $\Delta \mathcal{P}$, and the average excitation probability $\mathcal{E}$, given by

$$
\begin{aligned}
\mathcal{P} & =\hbar k \sum_{p^{\prime}} p^{\prime}\left|C_{p^{\prime}}\right|^{2} \\
\Delta \mathcal{P}^{2} & =\sum_{p^{\prime}}\left(\hbar k p^{\prime}-\mathcal{P}\right)^{2}\left|C_{p^{\prime}}\right|^{2} \\
\mathcal{E} & \left.\left.=\left|\sum_{p^{\prime}=\text { odd }} C_{p^{\prime}}\right| p^{\prime}\right\rangle\left.\right|^{2}=\left|\sum_{p=p^{\prime}=\text { odd }} C_{p^{\prime}} \mathrm{e}^{-\mathrm{i} p k \cdot R}\right| p\right\rangle\left.\right|^{2} \\
& =\sum_{p^{\prime}=\text { odd }}\left|C_{p^{\prime}}\right|^{2}
\end{aligned}
$$

where the replacement in the final stage of a coherent summation by an incoherent summation is allowed because of the broad spatial distribution. Since we assume that an atom left in the excited state will decay spontaneously before the next pulse pair, the total width of the momentum distribution will be given by

$\Delta \mathcal{P}_{\text {total }}^{2}=\Delta \mathcal{P}^{2}+(\hbar k)^{2} \mathcal{E}$

so that after $N$ pulse pairs, the momentum distribution will have a breadth given by $\Delta \mathcal{P}_{\text {1otal }} N^{1 / 2}$. This distribution is equivalent to heating to a temperature $T=\Delta \mathcal{P}_{\text {total }}^{2} N / 3 m k_{\mathrm{B}}$, and hence to a heating rate of $\Delta \mathcal{P}_{\text {total }}^{2} f / 2 m k_{\mathrm{B}}$ per unit time. The force on the atom is given by $\mathcal{P} f$.

Our calculated curves, showing the mean impulse $\mathcal{P}$ and the heating term $\Delta \mathcal{P}_{\text {total }}$ in units of $\hbar k$, as a function of displacement from the trap centre, are shown in Fig. 1. 

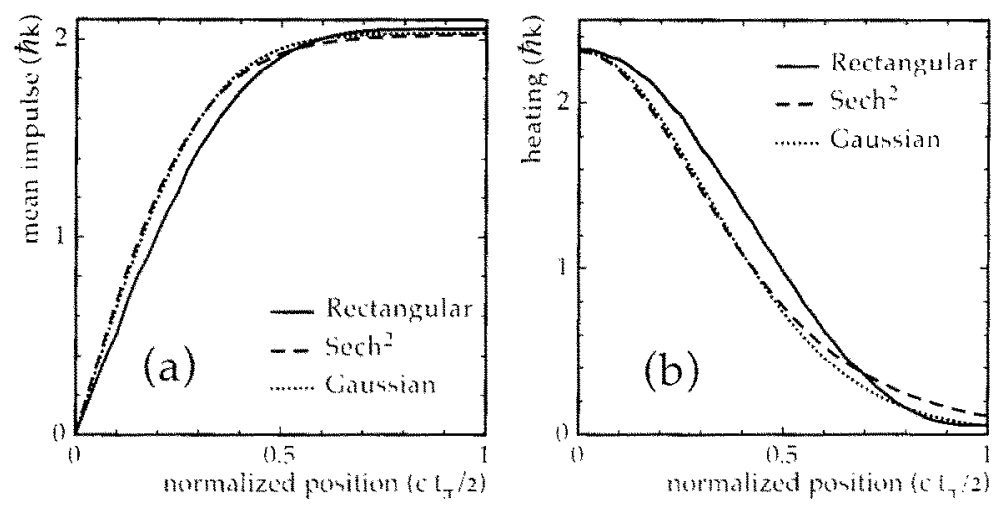

Fig. 1. (a) Mean impulse $\mathcal{P}$ and (b) the heating term $\Delta \mathcal{P}$ total in units of $\hbar k$, against normalized displacement from the trap centre measured in rectangular pulse half-widths, for three pulse shapes of equal energy. Accumulated errors from the numerical integration are around $2 \%$.

\section{Application}

The usual experimentally-convenient elements are also suitable candidates here: the alkali metals lithium, sodium, potassium, rubidium and caesium, together with barium and metastable helium and oxygen, all have strong transitions at accessible wavelengths. For sodium, for example [10], a 4 picosecond $\pi$-pulse has an intensity around $1 \mathrm{MW} \mathrm{cm}^{-2}$.

Although the process described thus far provides a restoring force in only one direction - along the laser beam axis - it may be extended to two dimensions by interleaving a second, perpendicular pair of pulses; the linear relation between force and displacement around the centre makes such an arrangement useful as a lens for atomic beams (although the performance will be affected by the heating within the pulse-overlap region ). Indeed, a third pulse pair may be added to provide three dimensional confinement, although because of the high intensities required, only relatively cold atoms will be contained within the small overlap of the three laser beams. It should be noted that, whilst the two pulses of a pair need not strictly counterpropagate but may simply converge, a geometry of three, triangularly-spaced (or four, tetrahedrally arranged) and simultaneous pulses will provide a force not towards the central point, but rather towards the three (six) lines of symmetry.

Radiation-pressure cooling may be readily applied to atoms confined in this way, for the two processes conflict only if the cooling laser saturates the trapping transition and thereby leaves population in the excited state; in this case, it will be necessary to turn off the cooling for a period prior to each pulse of the confining laser. If the transition is unsaturated, however, or if the cooling process uses a different atomic transition, no problem should occur. Similarly, if only a small fraction of the atomic distribution is saturated at any time, the average effect upon the confining process may be neglected.

The linear restoring force at the trap centre will cause slow atoms to oscillate with a characteristic frequency (typically $10 \mathrm{kHz}$ ). If the bulk displacement of the sample can be measured by monitoring the relative attenuation or amplification of the laser beams, then introducing a relative delay between the two beams should allow active control, or even stochastic cooling [11], of the sample.

An interesting variation is afforded by the phenomenon of adiabatic rapid passage, whereby chirped pulses invert the atomic population. The experimental arrangement in this case will resemble that which Nebenzahl [12] has suggested for isotope-selective deflection, but the pulses will be short and there should be a delay at the sample of one pulse period between the pulse and its reflection.

Current atom traps tend to be shallow but can confine atomic samples at ever lower temperatures. In contrast, the confining force described in this paper extends along the axis of the laser beams, yielding an extremely deep potential, but produces significant heating right at the trap centre. Whereas conventional trapping schemes are used for precision atomic spectroscopy, the force we describe might be of greater 
interest for focussing atomic or molecular beams, or even for manipulating free quasi-particles in the solidstate. Where most traps require narrow-band lasers and sharp atomic transitions, our scheme is intrinsically broad-band. Significant laser pulse energies are required, but the proposed scheme allows the confinement of neutral species without the application of electrostatic or magnetic fields.

We acknowledge with gratitude many discussions with past and present colleagues at the MPI für Quantenoptik. T.G.M.F. has been the grateful recipient of a bursary under the European Community SCIENCE programme.

\section{References}

[1] D.J. Wineland, W.M. Itano, Phys. Rev. A 20 (1979) 1521; J. Opt. Soc. Am B 6 (11) (1989) special issue.

[2| W. Paul, Rev. Mod. Phys. 62 (1990) 531.
[3] A. Ashkin, J.P. Gordon, Optics Lett. 8 (1983) 511.

[4] E.L. Raab, M. Prentiss, A. Cable, S. Chu, D.E. Pritchard, Phys. Rev. Lett. 59 (1987) 2631.

[5] P. Bouyer, P. Lemonde, M. Ben Dahan, A. Michaud, C. Salomon, J, Dalibard, Europhys. Lett. 27 (1994) 569.

[6] S. Chu, J.E. Bjorkholm, A. Ashkin, A. Cable, Phys. Rev. Lett. 57 (1986) 314.

[7] II. Friedmann, A.D. Wilson, Appl. Phys, Lett. 28 (1976) 270;

A.J. Palmer, J.F. Lam, J. Opt. Soc. Am B 3 (1986) 719; M. Allegrini, E. Arimondo, Phys. Lett. A 172 (1993) 271.

[8] C. Tanguy, S. Reynaud, C. Cohen-Tannoudji, J. Phys. B 17 (1984) 4623;

P.E. Moskowitz, P.L. Gould, D.E. Pritchard, J. Opt. Soc. Am B 2 (1985) 1784; Appl. Phys. B 54 (1992) 319.

[9] For example, R. Loudon. The quantum theory of light (OUP, Oxford, 1983).

[10] A.A. Radzig, B.M. Smimov, Reference data on atoms, molecules and ions, Springer series in chemical physics 31 (Springer, Berlin, 1985).

[11] N. Beverini et al, Phys. Rev. A 38 (1988) 107; Physica Scripta T 22 (1988) 238.

[12] l. Nebenzahl, A. Szöke, Appl. Phys. Lett. 25 (1974) 327 